\title{
28 THE UNDER-REPORT ADJUSTMENT OF INJURY DEATHS DATA FROM NATIONAL DISEASE SURVEILLANCE POINTS SYSTEM OF CHINA
}

doi:10.1136/injuryprev-2012-040590w.28

${ }^{1} \mathrm{Q}$ Li, ${ }^{2} \mathrm{M}$ Zhou, ${ }^{1} \mathrm{D}$ Bishai, ${ }^{2} \mathrm{~L}$ Wang, ${ }^{1} \mathrm{~S}$ Ma, ${ }^{1} \mathrm{AA}$ Hyder. ${ }^{1}$ Johns Hopkins Bloomberg School of Public Health, USA; ${ }^{2}$ Chinese Center for Disease Control and Prevention, China

Background The National Disease Surveillance Points System (DSPs) of China was established in 1980, and currently consists of 161 nationally representative urban and rural sites from 31 provinces covering 78 million people, a roughly $6 \%$ of Chinese population. To estimate the completeness of DSPs data, an independent household investigation of missing were conducted in 2009.

Aims/Objectives/Purpose To adjust the underreporting of injury death data in DSPs system.

Methods This paper proposes and compares two methods to correct for underreporting of injury deaths. First a cell-based weighting system is applied whereby underreporting rates for 18 cells defined by 3 age groups $\times 2$ sexes $\times 3$ regions were computed using the household survey as a gold standard. The second method is a propensity weighting method based on a logistic regression of underreport against age, sex, region, season, education, occupation, place of death and cause of death.

Results/Outcome The results from both adjustment methods performed better than the original DSPs data, compared with estimates from other sources such as WHO. The Life Expectancy at age 5 is 72.1 years in unadjusted data while compartment method and propensity weighting method respectively give 70.0 and 69.8, much closer to WHO estimate 70.3.

Significance/Contribution to the Field The adjusted DSPs data could provide valuable information for evidence-based injury-related policy-making in China. Additionally, the two approaches compared here can be applicable for other developing countries that also suffer underreporting problems. 